\title{
Introduction to controllability of non-linear systems
}

\author{
Ugo Boscain \\ CNRS, Laboratoire Jacques-Louis Lions, Sorbonne Université, team Inria CAGE \\ Mario Sigalotti \\ team Inria CAGE, Laboratoire Jacques-Louis Lions, Sorbonne Université
}

December 20, 2019

\begin{abstract}
We present some basic facts about the controllability of nonlinear finite dimensional systems. We introduce the concepts of Lie bracket and of Lie algebra generated by a family of vector fields. We then prove the Krener theorem on local accessibility and the Chow-Rashevskii theorem on controllability of symmetric systems. We then introduce the theory of compatible vector fields and we apply it to study control-affine systems with a recurrent drift or satisfying the strong Lie bracket generating assumption. We conclude with a general discussion about the orbit theorem by Sussmann and Nagano.
\end{abstract}

In this note we present some classical techniques to study the controllability of nonlinear systems. The discussion is kept as elementary as possible. Classical textbooks are $[1,2,3]$.

Consider the nonlinear control system

$$
\dot{x}=F(x, u(t)) \text {. }
$$

Here $x \in \mathbb{R}^{n}$ is the state of the system and $u(\cdot):\left[0, \infty\left[\rightarrow U \subset \mathbb{R}^{m}\right.\right.$ is the control.

We assume that $F$ is a $\operatorname{smooth}^{1}$ function of its arguments and that $u(\cdot)$ is regular enough in such a way that equation (1) with the initial condition $x(0)=x_{0} \in \mathbb{R}^{n}$ has local existence and uniqueness of solutions. For instance we can assume that $u(\cdot)$ is a $L_{\text {loc }}^{1}$ or a $L_{\text {loc }}^{\infty}$ function of the time. However, as it will be clear later, all conditions for controllability that we will get are actually sufficient conditions that are valid in the smaller class of piecewise constant controls.

Remark 1. For simplicity of notation in the following we also assume that for every $u(\cdot)$ a solution of (1) exists in $[0, \infty[$. However this hypothesis is not necessary for the validity of the theorems that we are going to prove.

Remark 2. In the discussion presented here we assume $x \in \mathbb{R}^{n}$. However almost nothing changes if we assume that $x$ belongs to a smooth connected manifold $M$ and that solutions of (1) exists in $[0, \infty[$. To extend the theory to smooth connected manifolds, if some non-trivial modification is necessary, we explicitly state it in the text.

\footnotetext{
${ }^{1}$ in this note by smooth we mean $\mathcal{C}^{\infty}$
} 
Denote by $x\left(t ; x_{0}, u(\cdot)\right)$ the solution at time $t$ of (1) starting from $x_{0}$ at $t=0$ and corresponding to a control function $u(\cdot)$. We recall the definitions of the reachable (or attainable) sets starting from $x_{0}$ :

- the reachable set from $x_{0}$ at time $\tau \geq 0$ is

$$
\mathcal{A}\left(\tau, x_{0}\right)=\left\{x_{1} \in \mathbb{R}^{n} \mid \exists u(\cdot):[0, \tau] \rightarrow U, x\left(\tau ; x_{0}, u(\cdot)\right)=x_{1}\right\} ;
$$

- the reachable set from $x_{0}$ within time $\tau \geq 0$ is

$$
\mathcal{A}\left(\leq \tau, x_{0}\right)=\cup_{t \in[0, \tau]} \mathcal{A}\left(t, x_{0}\right)
$$

- the reachable set from $x_{0}$ is

$$
\mathcal{A}\left(x_{0}\right)=\cup_{t \in[0,+\infty} \mathcal{A}\left(t, x_{0}\right)
$$

Given the control system (1), the purpose of the controllability theory is to characterize when these sets coincide with the entire state space.

Definition 3. The system (1) is said to be

- controllable if for every $x_{0} \in \mathbb{R}^{n}, \mathcal{A}\left(x_{0}\right)=\mathbb{R}^{n}$;

- small-time controllable if for every $x_{0} \in \mathbb{R}^{n}$ and $\tau>0$, we have $\mathcal{A}\left(\leq \tau, x_{0}\right)=\mathbb{R}^{n}$;

- small-time locally controllable at $x_{0}$ if $x_{0}$ belongs to the interior of $\mathcal{A}\left(\leq \tau, x_{0}\right)$ for every $\tau>0$.

\section{Control systems as families of vector fields}

In the following it will be useful to think to the system (1) as a family of vector fields ${ }^{2}$ parameterized by $u \in U$ (i.e., by constant controls). In other words we will often consider instead of the control system (1), the family of vector fields

$$
\mathcal{F}=\{F(\cdot, v) \mid v \in U\} .
$$

All vector fields of the family $\mathcal{F}$ are considered smooth and complete, i.e., for every $F \in \mathcal{F}$, $x_{0} \in \mathbb{R}^{n}$, the equation $\dot{x}=F(x)$ with initial condition $x(0)=x_{0}$ admits a solution in $]-\infty, \infty[$.

\section{1 $\operatorname{Vec}\left(\mathbb{R}^{n}\right)$ and its Lie algebra}

Let $\operatorname{Vec}\left(\mathbb{R}^{n}\right)$ be the vector space of all smooth vector fields in $\mathbb{R}^{n}$. Given a compete vector field $f \in \operatorname{Vec}\left(\mathbb{R}^{n}\right)$, let us indicate by $e^{t f}$ its flow, i.e., the map that with $x_{0}$ associates the solution at time $t$ to the Cauchy problem

$$
\left\{\begin{array}{l}
\dot{x}=f(x) \\
x(0)=x_{0}
\end{array}\right.
$$

\footnotetext{
${ }^{2}$ In an autonomous differential equation $\dot{x}=f(x)$, usually $f$ it is called a vector field, since it is a map that with every position $x$ associates a velocity vector $f(x)$.
} 
Although $e^{t f}$ is not an exponential in the usual sense, this notation is useful thanks to the following properties that are a direct consequence of existence and uniqueness of solutions of (2) and of their differentiability w.r.t. $x_{0}$ :

- for every $t, s \in \mathbb{R}, e^{(t+s) f}=e^{t f} \circ e^{s f}$;

- for every $t \in \mathbb{R}, e^{t f}$ is a diffeomorphism. In particular, it is invertible and we have $\left(e^{t f}\right)^{-1}=e^{-t f}$

- for every $t \in \mathbb{R},\left(\frac{d}{d t} e^{t f}\right)(x)=f\left(e^{t f} x\right)$. In particular, $\left(\left.\frac{d}{d t}\right|_{t=0} e^{t f}\right)(x)=f(x)$.

A crucial object in studying the controllability of (1) is the Lie algebra generated by the vector fields of the corresponding family $\mathcal{F}$. Let us first define the Lie bracket between two vector fields $f$ and $g$, as the vector field defined by

$$
[f, g](x)=D g(x) f(x)-D f(x) g(x) .
$$

Here, given a vector field $f=\left(f_{1}, \ldots, f_{n}\right)^{T}, D f$ is the matrix of partial derivatives of the components of $f$, i.e.,

$$
D f=\left(\begin{array}{ccc}
\partial_{1} f_{1} & \ldots & \partial_{n} f_{1} \\
\vdots & \ldots & \vdots \\
\partial_{1} f_{n} & \ldots & \partial_{n} f_{n}
\end{array}\right)
$$

One immediately verifies the following properties of the Lie bracket:

- bilinearity: for every $\lambda_{1}, \lambda_{2} \in \mathbb{R}$,

$$
\begin{aligned}
& {\left[f, \lambda_{1} g_{1}+\lambda_{2} g_{2}\right]=\lambda_{1}\left[f, g_{1}\right]+\lambda_{2}\left[f, g_{2}\right],} \\
& {\left[\lambda_{1} f_{1}+\lambda_{2} f_{2}, g\right]=\lambda_{1}\left[f_{1}, g\right]+\lambda_{2}\left[f_{2}, g\right] ;}
\end{aligned}
$$

- antisymmetry: $[g, f]=-[f, g]$;

- Jacobi identity: $[f,[g, h]]+[h,[f, g]]+[g,[h, f]]=0$.

A vector space $V$ endowed with an operation $V \times V \rightarrow V$ that is bilinear, antisymmetric and satisfying the Jacoby identity is said to be a Lie algebra. It follows that $\left(\operatorname{Vec}\left(\mathbb{R}^{n}\right),[\cdot, \cdot]\right)$ is a Lie algebra.

Remark 4. As a consequence of the antisymmetry of the Lie bracket, we have $[f, f]=0$. Notice moreover that the value of $[f, g]$ at a point $x$ does not depend only on the values of $f$ and $g$ at $x$, but also on their first order expansion at $x$. However, if at $x_{0}$ we have $f\left(x_{0}\right)=g\left(x_{0}\right)=0$, it follows from the definition that $[f, g]\left(x_{0}\right)=0$.

Example 5. Given two linear vector fields $A x$ and $B x$, where $A, B \in \mathbb{R}^{n \times n}$, we have that $[A x, B x]=B A x-A B x=-[A, B] x$.

The following lemma clarifies the geometric meaning of the Lie bracket: $[f, g]$ is a measure of the lack of commutation of the flows associated with $f$ and $g$. We refer to Figure 1 . 


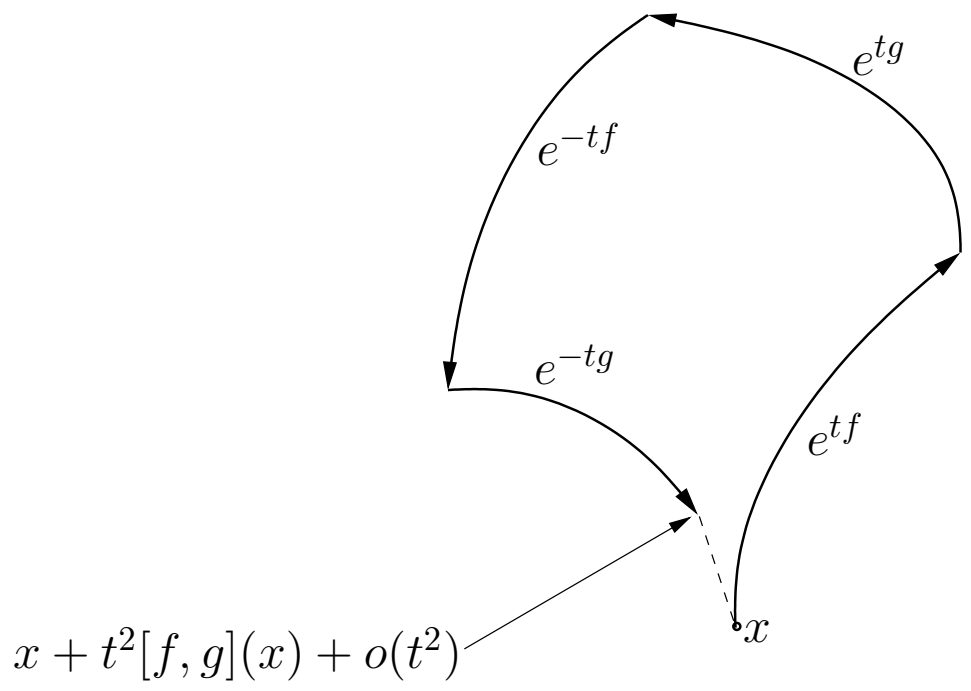

Figure 1: geometric meaning of the Lie bracket.

Lemma 6. For every $x \in \mathbb{R}^{n}$,

$$
e^{-t g} \circ e^{-t f} \circ e^{t g} \circ e^{t f}(x)=x+t^{2}[f, g](x)+O\left(t^{3}\right),
$$

for t that tends to zero.

Proof. It is enough to compute for each flow the Taylor expansion at order 3 . We have

$$
e^{t f}(x)=x+t f(x)+\frac{t^{2}}{2} D f(x) f(x)+O\left(t^{3}\right),
$$

and

$$
e^{t g} \circ e^{t f}(x)=x+t(f(x)+g(x))+\frac{t^{2}}{2} D f(x) f(x)+t^{2} D g(x) f(x)+\frac{t^{2}}{2} D g(x) g(x)+O\left(t^{3}\right) .
$$

Then

$$
e^{-t f} \circ e^{t g} \circ e^{t f}(x)=x+t g(x)+[f, g](x) t^{2}+\frac{t^{2}}{2} D g(x) g(x)+O\left(t^{3}\right) .
$$

At the next step the result follows.

Remark 7. The previous lemma says in particular that if $[f, g](x) \notin \operatorname{Vect}(f(x), g(x))$, then it is possible, by alternating between the dynamics of $f$ and $g$, to attain points that cannot be reached with the flow of linear combinations of $f$ and $g$. This is the starting idea behind the conditions for controllability that we are going to study in this note. Notice however that in order to generate the Lie bracket $[f, g]$, one needs to be able to use, beside $f$ and $g$, also $-f$ and $-g$, otherwise one is much more constrained in the possible movements. As a consequence, it will be easier to prove controllability results for symmetric systems (i.e., systems for which if $f \in \mathcal{F}$ then $-f \in \mathcal{F}$ ). See Section 3 .

An important corollary of the previous lemma is the following.

Corollary 8. The flows $e^{t f}$ and $e^{t g}$ (corresponding to the vector fields $f$ and $g$ ) commute for every $t \in \mathbb{R}$ if and only if their Lie bracket $[f, g](x)=0$ for every $x \in \mathbb{R}^{n}$. 
Proof. The fact that commutation of the flows implies that the Lie bracket vanishes follows immediately from (4). Concerning the converse implication, let us consider the curve

$$
\gamma(s)=e^{-\sqrt{s} g} \circ e^{-\sqrt{s} f} \circ e^{\sqrt{s} g} \circ e^{\sqrt{s} f}(x) .
$$

By Lemma 6, we have that $\gamma$ is differentiable at each time $s$ and

$$
\frac{d \gamma}{d s}=[f, g](\gamma(s))
$$

If $[f, g]$ is identically equal to zero, $\gamma$ turns out to be solution of

$$
\frac{d \gamma}{d s}=0, \quad \gamma(0)=0
$$

It follows $\gamma(s)=0$ for every $s$ and $e^{\sqrt{s} g} \circ e^{\sqrt{s} f}=e^{\sqrt{s} g} \circ e^{\sqrt{s} f}$. Setting $t=\sqrt{s}$ the result follows.

Remark 9. In a differentiable manifold, Definition 3 and formula (4) make sense only in coordinates. An intrinsic definition of Lie bracket is

$$
[f, g](x)=\left.\frac{d}{d s}\right|_{s=0} e^{-\sqrt{s} g} \circ e^{-\sqrt{s} f} \circ e^{\sqrt{s} g} \circ e^{\sqrt{s} f}(x) .
$$

Definition 10. Let $\mathcal{F}$ be a family of vector fields. We call Lie $(\mathcal{F})$ the smallest sub-algebra of $\operatorname{Vec}\left(\mathbb{R}^{n}\right)$ containing $\mathcal{F}$. Namely, $\operatorname{Lie}(\mathcal{F})$ is the span of all vector fields of $\mathcal{F}$ and of their iterated Lie brackets of any order:

$$
\operatorname{Lie}(\mathcal{F})=\operatorname{span}\left\{f_{1},\left[f_{1}, f_{2}\right],\left[f_{1},\left[f_{2}, f_{3}\right]\right],\left[f_{1},\left[f_{2},\left[f_{3}, f_{4}\right]\right]\right], \ldots \mid f_{1}, f_{2}, \ldots \in \mathcal{F}\right\} .
$$

Definition 11. We say that the family $\mathcal{F}$ is Lie bracket generating at a point $x$ if the dimension of $\operatorname{Lie}_{x}(\mathcal{F}):=\{f(x) \mid f \in \operatorname{Lie}(\mathcal{F})\}$ is equal to $n$. We say that the family $\mathcal{F}$ is Lie bracket generating if this condition is verified for every $x \in \mathbb{R}^{n}$.

Remark 12. Notice that in general $\operatorname{Lie}(\mathcal{F})$ is an infinite-dimensional space, while $\operatorname{Lie}_{x}(\mathcal{F})$ is a subspace of $\mathbb{R}^{n}$.

Exercise 1. Let $\mathcal{F}=\left\{f_{1}, \ldots, f_{m}\right\}$ and let $A$ be an invertible $m \times m$ matrix. Define $f_{i}^{\prime}=$ $\sum_{j} A_{i j} f_{j}$ and $\mathcal{F}^{\prime}=\left\{f_{1}^{\prime}, \ldots, f_{m}^{\prime}\right\}$. Prove that $\mathcal{F}$ is Lie bracket generating if and only if $\mathcal{F}^{\prime}$ is.

\subsection{Affine control systems}

An affine control system is a system of the form

$$
\dot{x}=f_{0}(x)+\sum_{i=1}^{m} u_{i}(t) f_{i}(x),
$$

where $f_{0}, f_{1}, \ldots, f_{m}$ belong to $\operatorname{Vec}\left(\mathbb{R}^{n}\right)$ and $u(\cdot)=\left(u_{1}(\cdot), \ldots, u_{m}(\cdot)\right):\left[0, \infty\left[\rightarrow U \subset \mathbb{R}^{m}\right.\right.$ is the control. In an affine control system it is also assumed that $U$ contains a neighborhood of the origin in $\mathbb{R}^{m}$. The vector fields $f_{0}$ is called the drift.

Exercise 2. Let $\mathcal{F}$ be the family of vector fields associated with (5).

- Prove that if $\left\{f_{0}, f_{1}, \ldots, f_{m}\right\}$ is Lie bracket generating then also $\mathcal{F}$ is.

- Prove that if $\left\{f_{1}, \ldots, f_{m}\right\}$ is Lie bracket generating then also $\mathcal{F}$ is. 

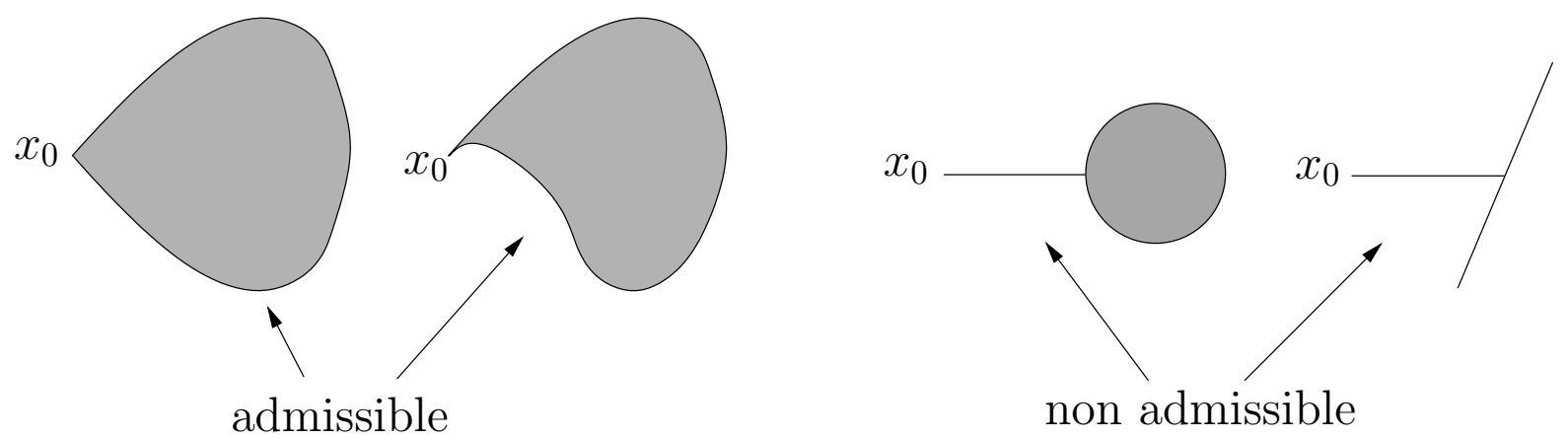

Figure 2: admissible and non-admissible reachable sets when the system is Lie bracket generating at $x_{0}$.

\section{The Krener Theorem: local accessibility}

The fact that a control system is Lie bracket generating does not permit in general to conclude that it is controllable. Consider for instance the control system on the plane

$$
\left(\begin{array}{c}
\dot{x}_{1} \\
\dot{x}_{2}
\end{array}\right)=\left(\begin{array}{l}
1 \\
0
\end{array}\right)+u(t)\left(\begin{array}{l}
0 \\
1
\end{array}\right)
$$

where $u(\cdot):[0, \infty[\rightarrow[-1,1]$. It is Lie bracket generating (since the corresponding family $\mathcal{F}$ contains the vector fields $\{(1,1),(1,-1)\}$, however starting from the origin one cannot reach any point whose first coordinate is negative. This is essentially due to the fact that the family $\mathcal{F}$ contains two vector fields but not their opposite (cf. Remark 7). The Lie bracket generating condition permits to say that a system is locally accessible in the following sense.

Theorem 13 (Krener). If $\mathcal{F}$ is Lie bracket generating at $x_{0}$, then for every $\tau>0, x_{0}$ belongs to the closure of the interior of $\mathcal{A}\left(\leq \tau, x_{0}\right)$.

The conclusion of the Krener Theorem can be equivalently reformulated in the following way:

- for every $\tau>0$, the set $\mathcal{A}\left(\leq \tau, x_{0}\right)$ has nonempty interior,

- $x_{0}$ is a density point of such an interior.

Krener's theorem says in particular that the trajectories starting from a point at which the system is Lie bracket generating can reach (in an arbitrarily small time) a set having nonempty interior. Figure 2 shows what one can expect/non-expect from $\mathcal{A}\left(\leq \tau, x_{0}\right), \tau>0$.

Proof of Theorem 13. First notice that if $\mathcal{F}$ is Lie bracket generating at a point, then the same property holds true in a neighborhood of that point. (If $n$ vector fields are linearly independent at a point, then they are linearly independent in a neighborhood of that point.)

There exists $f \in \mathcal{F}$ such that $f\left(x_{0}\right) \neq 0$, otherwise $\operatorname{Lie}_{x_{0}}(\mathcal{F})=\{0\}$. If $n=1$ the conclusion follows.

If $n>1$ and all vector fields of $\mathcal{F}$ are tangent to the curve $t \mapsto e^{t f}\left(x_{0}\right), 0<t<\varepsilon$, then from Lemma 6 it follows that $\operatorname{Lie}_{e^{t f}\left(x_{0}\right)}(\mathcal{F})$ is also tangent to that curve and hence its dimension is less than 2. This contradicts the Lie bracket generating assumption. As a consequence, there 


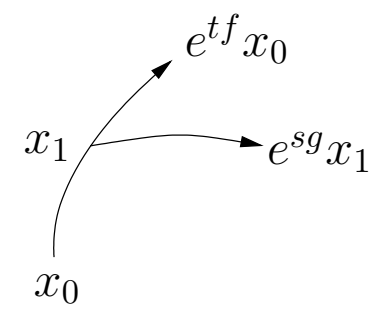

Figure 3: Proof of Krener Theorem

exist $g \in \mathcal{F}$ and $0<\bar{t}<\varepsilon$ such that $f$ and $g$ are linearly independent in a neighborhood of $x_{1}=e^{\bar{t} f}\left(x_{0}\right)$ (see Figure 3). Hence $(t, s) \mapsto e^{s g} \circ e^{t f}\left(x_{0}\right), 0<s<\varepsilon^{\prime}, \bar{t}-\varepsilon^{\prime}<t<\bar{t}+\varepsilon^{\prime}$ has as image a surface of dimension 2. If $n=2$ the conlcusion follows.

Otherwise we iterate the same argument and we conclude by recurrence on $n$.

Remark 14. Notice that for this proof we have only used piecewise constant controls.

Remark 15. From the proof of the Krener theorem it follows that $\mathcal{A}\left(\leq \tau, x_{0}\right), \tau>0$, contains an open set $\Omega$ having $x_{0}$ in its closure whose points can be reached by trajectories of the type $e^{t_{n} f_{i_{n}}} \circ \ldots \circ e^{t_{1} f_{i_{1}}} x_{0}$ where $t_{1}, \ldots, t_{n}>0$ and $f_{i_{1}}, \ldots, f_{i_{n}} \in \mathcal{F}$, i.e., by trajectories corresponding to piecewise constant controls made by $n$ pieces. Notice that the vector fields $f_{i_{1}}, \ldots, f_{i_{n}}$ could be repeated. For instance if $\mathcal{F}=\left\{f_{1}, f_{2}\right\}$ is a Lie bracket generating family and we are in dimension 3, we could have for instance $f_{i_{1}}=f_{1}, f_{i_{2}}=f_{2}, f_{i_{3}}=f_{1}$.

\section{Symmetric systems}

Definition 16. A family of vector fields $\mathcal{F}$ is said to be symmetric if $f \in \mathcal{F}$ implies $-f \in \mathcal{F}$.

When the family $\mathcal{F}$ is Lie bracket generating and symmetric one obtain that the system is controllable. This is the conclusion of the celebrated Chow-Rashevskii theorem.

Theorem 17 (Chow-Rashevskii). If $\mathcal{F}$ is Lie bracket generating and symmetric, then for every $x_{0} \in \mathbb{R}^{n}$ we have $\mathcal{A}\left(x_{0}\right)=\mathbb{R}^{n}$.

Proof. Step 1. Fix $x_{0} \in \mathbb{R}^{n}$ and let us show that $\mathcal{A}\left(x_{0}\right)$ contains a neighborhood of $x_{0}$. Since $\mathcal{F}$ is Lie bracket generating, $\mathcal{A}\left(x_{0}\right)$ contains a nonempty open set $\Omega$ whose points can be reached by trajectories corresponding to piecewise controls made by $n$ pieces. Fix $t_{1}, \ldots, t_{n}>$ 0 and $f_{i_{1}}, \ldots, f_{i_{n}} \in \mathcal{F}$ such that $\bar{x}_{0}:=e^{t_{n} f_{i_{n}}} \circ \ldots \circ e^{t_{1} f_{i_{1}}} x_{0} \in \Omega$. Since $\mathcal{F}$ is symmetric, $-f_{i_{1}}, \ldots,-f_{i_{n}} \in \mathcal{F}$ and we have that

$$
e^{-t_{1} f_{i_{1}}} \circ \ldots \circ e^{-t_{n} f_{i_{n}}}\left(\mathcal{A}\left(x_{0}\right)\right) \subset \mathcal{A}\left(x_{0}\right) .
$$

In particular $\mathcal{A}\left(x_{0}\right)$ contains the set

$$
V=e^{-t_{1} f_{i_{1}}} \circ \ldots \circ e^{-t_{n} f_{i_{n}}}(\Omega) .
$$

Now, since $\Omega$ is open and $e^{-t_{1} f_{i_{1}}} \circ \ldots \circ e^{-t_{n} f_{i_{n}}}$ is a diffeomorphism, we have that $V$ is open. Moreover $V$ contains $x_{0}$ since $\bar{x}_{0} \in \Omega$ and $e^{-t_{1} f_{i_{1}}} \circ \ldots \circ e^{-t_{n} f_{i_{n}}} \bar{x}_{0}=x_{0}$. It follows that, for every $x_{0}, \mathcal{A}\left(x_{0}\right)$ contains a neighborhood of $x_{0}$. 
Step 2. Let us show that $\mathcal{A}\left(x_{0}\right)$ is open. If $x_{1} \in \mathcal{A}\left(x_{0}\right)$, then $\mathcal{A}\left(x_{1}\right) \subset \mathcal{A}\left(x_{0}\right)$. It follows that $\operatorname{int}\left(\mathcal{A}\left(x_{1}\right)\right) \subset \operatorname{int}\left(\mathcal{A}\left(x_{0}\right)\right)$. But from Step 1 we have that $x_{1} \in \operatorname{int}\left(\mathcal{A}\left(x_{1}\right)\right)$. Hence $x_{1} \in$ $\operatorname{int}\left(\mathcal{A}\left(x_{0}\right)\right)$.

Step 3. From the fact that $\mathcal{F}$ is symmetric, it follows that $x_{1} \in \mathcal{A}\left(x_{0}\right)$ if and only if $x_{0} \in \mathcal{A}\left(x_{1}\right)$. Let us consider the equivalence classes $\mathbb{R}^{n} / \sim$ where $\sim$ is the equivalence relation

$$
x_{1} \sim x_{0} \text { if and only if } x_{1} \in \mathcal{A}\left(x_{0}\right) .
$$

Such equivalence classes are open and disjoint. Since $\mathbb{R}^{n}$ is connected, it follows that there is only one class. Hence, for every $x_{0}$ we have $\mathcal{A}\left(x_{0}\right)=\mathbb{R}^{n}$.

Remark 18. Notice that in Step 1 of the proof of Chow-Rashevskii theorem, since the times can be rendered arbitrarily small, we have proved that the system is small-time locally controllable in a neighborhood of every point $x_{0}$. Also, we have proved that every point of a neighborhood of $x_{0}$ can be reached with trajectories made by $2 n$ pieces.

Remark 19. Notice that the Chow-Rashevskii theorem can be used in more general situations than those fixed by the hypotheses stated here. For instance

- to get controllability it is sufficient that the family $\mathcal{F}$ contains a symmetric family of Lie bracket generating vector fields;

- if one can prove that $\mathcal{F}$ is symmetric and Lie bracket generating in a connected open set $U$ of $\mathbb{R}^{n}$ then one get the system is controllable in $U$.

\section{Compatible vector fields}

When a family of vector fields is Lie bracket generating but is not symmetric, in general it is not easy to understand if the system is controllable or not. A technique to study the controllability is the one of compatible vector fields.

Definition 20. A vector field $g$ is said to be compatible with the family $\mathcal{F}$ if defining $\hat{\mathcal{F}}=$ $\mathcal{F} \cup\{g\}$ we have the following: For every $x_{0} \in \mathbb{R}^{n}$, the reachable set $\hat{\mathcal{A}}\left(x_{0}\right)$ of $\hat{\mathcal{F}}$ is contained in the closure of $\mathcal{A}\left(x_{0}\right)$.

The main result of the theory of compatible vector fields is the following.

Theorem 21. If $\mathcal{F}$ is a Lie bracket generating family of vector fields, $g$ is compatible with $\mathcal{F}$ and $\mathcal{F} \cup\{g\}$ is controllable, then $\mathcal{F}$ is controllable as well.

This theorem should be used in the following way: one looks for a vector field $g$ that added to the family $\mathcal{F}$ do not change the closure of the reachable set and such that it is easy to prove the controllability of the family $\mathcal{F} \cup\{g\}$.

The main ingredient to prove Theorem 21 is the following corollary of the Krener theorem.

Corollary 22. If $\mathcal{F}$ is Lie bracket generating and $\mathcal{A}\left(x_{0}\right)$ is dense in $\mathbb{R}^{n}$ for some $x_{0}$, then $\mathcal{A}\left(x_{0}\right)=\mathbb{R}^{n}$. 


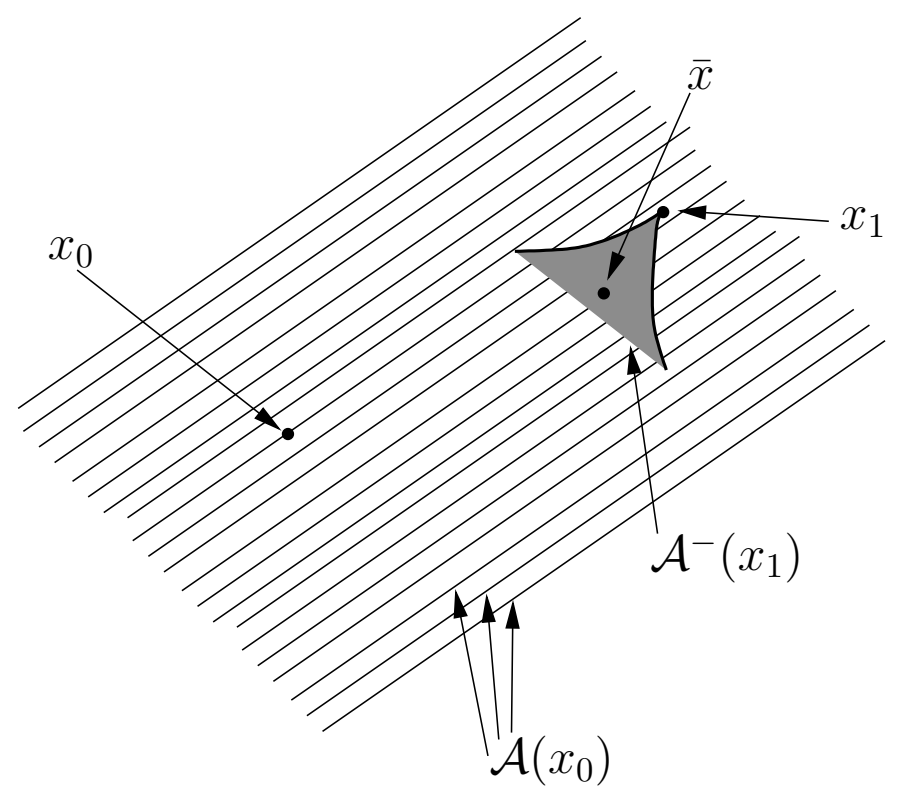

Figure 4: Proof of Corollary 22

Proof. Let $x_{1} \in \mathbb{R}^{n}$ and consider the system

$$
\dot{x}=-F(x, u(t)), \quad x \in \mathbb{R}^{n}, \quad u(\cdot):\left[0, \infty\left[\rightarrow U \subset \mathbb{R}^{m},\right.\right.
$$

which is obtained from (1) by reversing the time. Let $\mathcal{F}^{-}$be the family of vector fields associated with (6). Since $\mathcal{F}$ is Lie bracket generating, then $\mathcal{F}^{-}$is Lie bracket generating as well.

Let $\mathcal{A}^{-}\left(x_{1}\right)$ be the reachable set for (6) starting from $x_{1}$. Thanks to Krener's theorem, $\mathcal{A}^{-}\left(x_{1}\right)$ contains a nonempty open set. In particular it has nonempty intersection with $\mathcal{A}\left(x_{0}\right)$ (being $\mathcal{A}\left(x_{0}\right)$ dense). See Figure 4.

This means that $x_{1} \in \mathcal{A}\left(x_{0}\right)$. Indeed from $x_{0}$ one can reach a point $\bar{x} \in \mathcal{A}\left(x_{0}\right) \cap \mathcal{A}^{-}\left(x_{1}\right)$ (since $\left.\bar{x} \in \mathcal{A}\left(x_{0}\right)\right)$ and from $\bar{x}$ one can reach $x_{1}$ (since $\bar{x}$ is reachable from $x_{1}$ for the system with reverted time).

Being $x_{1}$ arbitrary we have that $\mathcal{A}\left(x_{0}\right)=\mathbb{R}^{n}$.

Proof of Theorem 21. Let $\mathcal{A}\left(x_{0}\right)$ be the reachable set from $x_{0}$ associated with $\mathcal{F}$ and $\hat{\mathcal{A}}\left(x_{0}\right)$ be the reachable set from $x_{0}$ associated with $\mathcal{F} \cup\{g\}$. For every $x_{0} \in \mathbb{R}^{n}$ we have $\mathbb{R}^{n}=\hat{\mathcal{A}}\left(x_{0}\right) \subset$ $\overline{\mathcal{A}}\left(x_{0}\right)$. Hence $\mathcal{A}\left(x_{0}\right)$ is dense. Since the system is Lie bracket generating, from Corollary 22 the conclusion follows.

Next we present some important applications of the technique based on compatible vector fields. 


\subsection{Affine systems with recurrent drift}

In this section we apply the theory of compatible vector fields to affine control systems (cf. Section 1.2) that are Lie bracket generating and having a drift $f_{0}$ which is weakly recurrent ${ }^{3}$.

Definition 24 (Weakly recurrent vector field). A vector field $f$ is said to be weakly recurrent if for every point $x_{0} \in \mathbb{R}^{n}$, every neighborhood $V$ of $x_{0}$ and every time $t>0$, there exist $\bar{x}_{0} \in V$ and $t^{*}>t$ such that $e^{t^{*} f}\left(\bar{x}_{0}\right) \in V$.

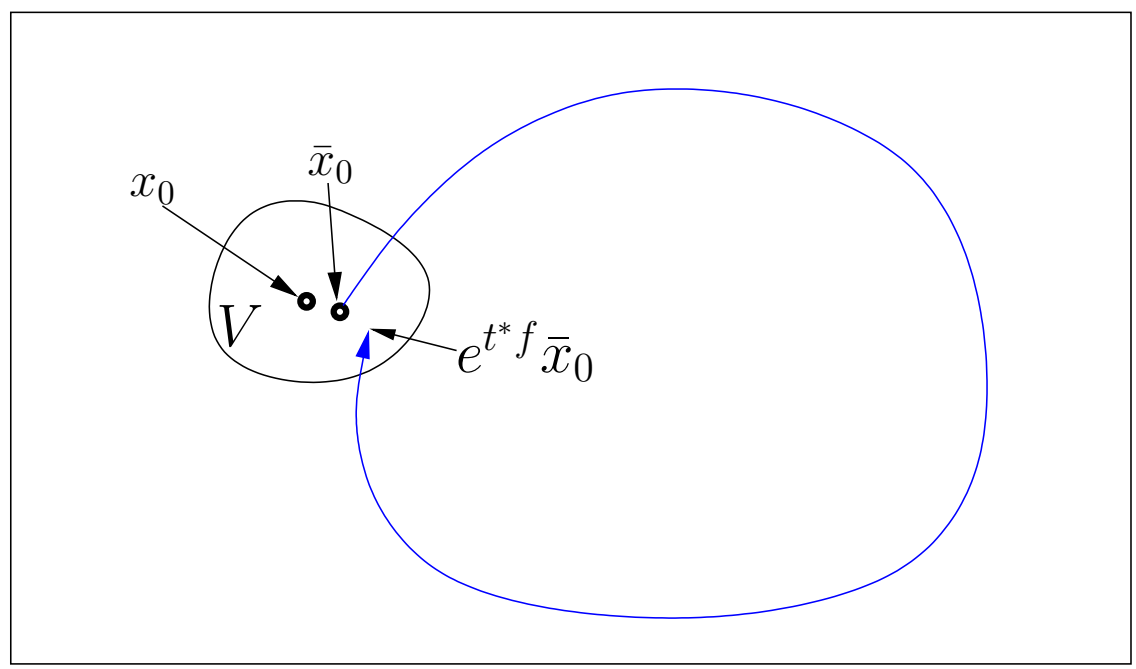

Notice that if the trajectories of $f$ are periodic (possibly with period depending on the trajectory), then $f$ is recurrent.

Lemma 25. Let $\mathcal{F}$ be a Lie bracket generating family of vector fields and $f \in \mathcal{F}$. If $f$ is weakly recurrent then $-f$ is compatible with $\mathcal{F}$.

Proof. We have to prove that for every $x_{0}$ and for every $t>0, e^{-t f} x_{0}$ can be obtained as limit of points belonging to the reachable set $\mathcal{A}\left(x_{0}\right)$.

By Krener theorem (thanks to the fact that $\mathcal{F}$ is Lie-bracket generating), there exists an arbitrarily small open set $W \subset \mathcal{A}\left(x_{0}\right)$ such that $x_{0} \in \bar{W}$.

Now since $e^{-t f}$ is a diffeomorphism this implies that $e^{-t f} x_{0} \in \overline{e^{-t f} W}$.

Since $f$ is weakly recurrent, there exists $t^{*}>t$ such that

$$
e^{t^{*} f} e^{-t f} W \cap e^{-t f} W \neq \emptyset
$$

or equivalently

$$
e^{\left(t^{*}-t\right) f} W \cap e^{-t f} W \neq \emptyset .
$$

But since $t^{*}-t>0$ and $W \subset \mathcal{A}\left(x_{0}\right)$ we have that $e^{\left(t^{*}-t\right) f} W \subset \mathcal{A}\left(x_{0}\right)$. It follows that

$$
\mathcal{A}\left(x_{0}\right) \cap e^{-t f} W \neq \emptyset .
$$

Hence in any neighborhood of $e^{-t f} x_{0}$ there are points of $\mathcal{A}\left(x_{0}\right)$. In other words $e^{-t f} x_{0} \in \overline{\mathcal{A}\left(x_{0}\right)}$.

\footnotetext{
${ }^{3}$ the term weakly recurrent comes from the fact that our definition is a bit more general than the classical definition of recurrent vector field:
}

Definition 23 (Recurrent vector field). A vector field $f$ is said to be recurrent if for every point $x_{0} \in \mathbb{R}^{n}$, every neighborhood $V$ of $x_{0}$ and every time $t>0$, there exists $t^{*}>t$ such that $e^{t^{*}} f\left(x_{0}\right) \in V$. 


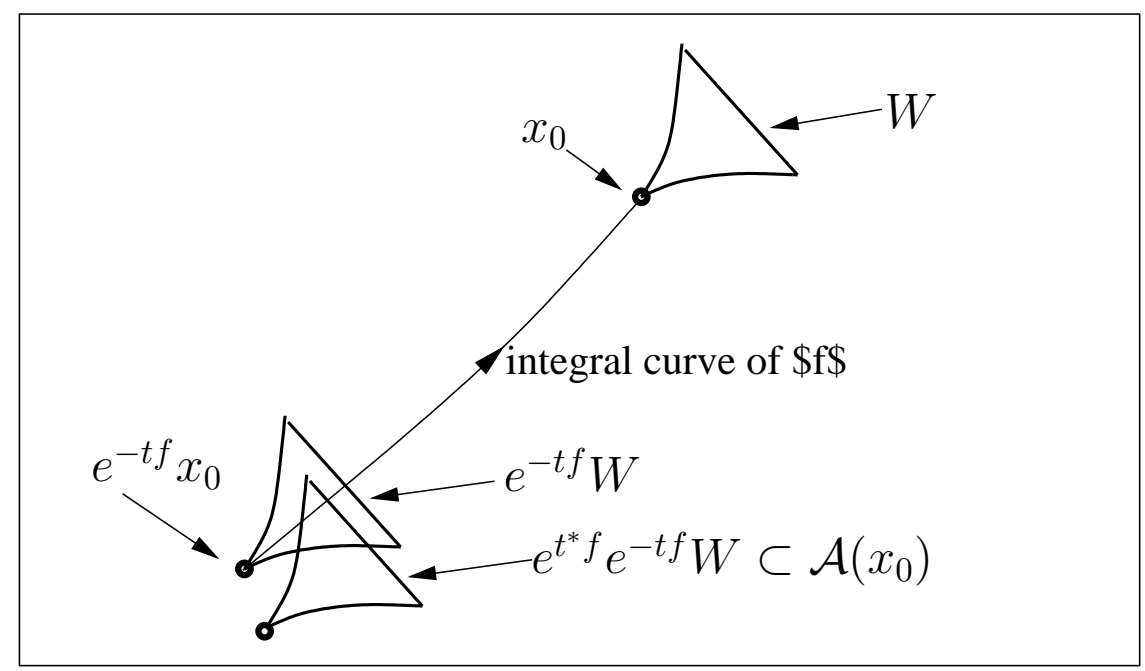

As a consequence of the previous lemma we have the following.

Corollary 26. Consider the control system

$$
\dot{x}=f_{0}(x)+\sum_{i=1}^{m} u_{i}(t) f_{i}(x), \quad\left(u_{1}(\cdot), \ldots, u_{m}(\cdot)\right):\left[0, \infty\left[\rightarrow U \subset \mathbf{R}^{m} .\right.\right.
$$

Assume that (i) 0 belongs to the interior of $U$, (ii) the control system (7) is Lie bracket generating, (iii) $f_{0}$ is weakly recurrent. Then the system is controllable.

Proof. Notice that $f_{0} \in \mathcal{F}$ since 0 belongs to $U$. Lemma 25 states the equivalence between the controllability of (7) and that of

$$
\dot{x}=\sum_{i=0}^{m} u_{i}(t) f_{i}(x), \quad\left(u_{0}(\cdot), \ldots, u_{m}(\cdot)\right):[0, \infty[\rightarrow\{-1,1\} \times U .
$$

The controllability of this system follows from the Chow-Rashevskii theorem since $\{-1,1\} \times U$ contains a symmetric set (Remark 19).

Example (on a manifold). Consider the control system on the sphere $S^{2}=\left\{x \in \mathbb{R}^{3} \mid\right.$ $\left.x_{1}^{2}+x_{2}^{2}+x_{3}^{2}=1\right\}$ given by

$$
\dot{x}=f_{0}(x)+u f_{1}(x), \quad u(\cdot):\left[0, \infty\left[\rightarrow(-1,1), \quad x \in S^{2},\right.\right.
$$

where

$$
f_{0}(x)=\left(\begin{array}{c}
-x_{2} \\
x_{1} \\
0
\end{array}\right), \quad f_{1}(x)=\left(\begin{array}{c}
0 \\
-x_{3} \\
x_{2}
\end{array}\right) .
$$

The flows of $f_{0}$ and $f_{1}$ are rotations around the axes $(0,0,1)^{T}$ and $(1,0,0)^{T}$, respectively.

This system is controllable since 
- the Lie bracket between $f_{0}$ and $f_{1}$ is given by

$$
\left[f_{0}, f_{1}\right](x)=\left(\begin{array}{c}
-x_{3} \\
0 \\
x_{1}
\end{array}\right)
$$

and hence the system is Lie bracket generating (for every $x \in S^{2}, \operatorname{dim}\left(\operatorname{span}\left\{f_{0}, f_{1}, f_{2}\right\}(x)=\right.$ $2)$;

- the trajectories of $f_{0}$ are periodic and hence $f_{0}$ is recurrent.

\subsection{Affine systems with non-recurrent drift}

When the drift is not recurrent one can still obtain that the system is controllable if the controls are unbounded and if it is not necessary to use the drift to get a Lie algebra of full dimension at every point. More precisely we have the following.

Proposition 27 (Strong bracket generating). Consider the control system

$$
\dot{x}=f_{0}(x)+\sum_{i=1}^{m} u_{i} f_{i}(x), \quad\left(u_{1}(\cdot), \ldots, u_{m}(\cdot)\right):\left[0, \infty\left[\rightarrow \mathbb{R}^{m} .\right.\right.
$$

If $\left\{f_{1}, \ldots, f_{m}\right\}$ is Lie bracket generating then (8) is controllable.

Remark 28. For an affine control system as (8), the condition that $\left\{f_{1}, \ldots, f_{m}\right\}$ is Lie bracket generating is called the strong bracket generating condition.

Proof. First notice that as a consequence of Exercise 2, being $\left\{f_{1}, \ldots, f_{m}\right\}$ Lie bracket generating, then (8) is Lie bracket generating as well. Let $\mathcal{F}$ be the family of vector fields associated with (8). In the following we are going to prove that for every $\left(v_{1}, \ldots, v_{m}\right) \in \mathbb{R}^{m}$ the vector field $\sum_{i=1}^{m} v_{i} f_{i}$ is compatible with $\mathcal{F}$. Once this is done, the controllability of (8) follows, since the family $\mathcal{F} \cup\left\{\sum_{i=1}^{m} v_{i} f_{i}, \quad v_{i} \in \mathbb{R}, i=1, \ldots, m\right\}$ contains a symmetric and Lie bracket generating sub-family (Remark 19).

To show that $\sum_{i=1}^{m} v_{i} f_{i}$ is compatible with $\mathcal{F}$ for every $v_{1}, \ldots, v_{m} \in \mathbb{R}$, remark that

$$
\sum_{i=1}^{m} v_{i} f_{i}=\lim _{n \rightarrow \infty} \frac{1}{n}\left(f_{0}+\sum_{i=1}^{m}\left(n v_{i}\right) f_{i}\right)
$$

and that $\frac{1}{n}\left(f_{0}+\sum_{i=1}^{m}\left(n v_{i}\right) f_{i}\right)$ is compatible with $\mathcal{F}$ since a trajectory of (8) corresponding to controls $\tilde{u}_{i}(\cdot), i=1, \ldots, m$, is a time-reparameterisation of a trajectory of

$$
\dot{x}=\frac{1}{n}\left(f_{0}(x)+\sum_{i=1}^{m} u_{i} f_{i}(x)\right)
$$

corresponding to controls $n \tilde{u}_{i}(\cdot), i=1, \ldots, m$.

The thesis follows from the fact that if a vector field is the uniform limit on all compacts of a sequence of compatible vector fields, then it is compatible as well (from the continuity of solutions of ODEs with respect to the vector field). 


\subsection{Convexification}

A very useful criterium is the one that states that a convex combination of vector fields of $\mathcal{F}$ is compatible with $\mathcal{F}$. It formalize the intuition that if one commutes quickly between the dynamics of two vector fields, and one stays the same time on each dynamics, then the corresponding trajectory is close the trajectory of $\frac{f+g}{2}$ starting from the same point.

Lemma 29. For every $\lambda_{1}, \ldots, \lambda_{k} \geq 0$ and $f_{1}, \ldots, f_{k} \in \mathcal{F}$, the vector field $\lambda_{1} f_{1}+\cdots+\lambda_{k} f_{k}$ is compatible with $\mathcal{F}$.

The proof of this Lemma is quite technical and it is based on the Gronwall inequality. See [1] for details.

From this lemma one can gets some useful corollaries of Theorem 17, Corollary 26, and Proposition 32.

Corollary 30. If $\mathcal{F}$ is Lie bracket generating and its convex hull in the space $\operatorname{Vec}\left(\mathbb{R}^{n}\right)$ is symmetric, then for every $x_{0} \in \mathbb{R}^{n}$ we have $\mathcal{A}\left(x_{0}\right)=\mathbb{R}^{n}$.

Corollary 31. Consider the control system

$$
\dot{x}=f_{0}(x)+\sum_{i=1}^{m} u_{i} f_{i}(x), \quad\left(u_{1}(\cdot), \ldots, u_{m}(\cdot)\right):\left[0, \infty\left[\rightarrow U \subset \mathbf{R}^{m} .\right.\right.
$$

Assume that (i) 0 belongs to the interior of the convex hull of $U$, (ii) the control system (10) is Lie bracket generating, (iii) $f_{0}$ is weakly recurrent. Then the system is controllable.

Corollary 32. Consider the control system

$$
\dot{x}=f_{0}(x)+\sum_{i=1}^{m} u_{i} f_{i}(x), \quad\left(u_{1}(\cdot), \ldots, u_{m}(\cdot)\right):\left[0, \infty\left[\rightarrow U \subset \mathbb{R}^{m} .\right.\right.
$$

If the convex hull of $U$ is $\mathbb{R}^{m}$ and if $\left\{f_{1}, \ldots, f_{m}\right\}$ is Lie bracket generating then (11) is controllable.

\section{$5 \quad$ Orbits and necessary conditions for controllability}

We have seen in the previous sections several sufficient conditions for the controllability of a nonlinear system. In this section we discuss some necessary conditions that are consequence of a very deep theorem of geometric nature, the so-called orbit theorem. This theorem permits to conclude that, beside pathological cases, $\operatorname{Lie}_{x}(\mathcal{F})$ measures precisely the dimension of the set of directions that can be used starting from a point.

We define the orbit of the family $\mathcal{F}$ starting from a point $x_{0} \in \mathbb{R}^{n}$ as the set

$$
\mathcal{O}\left(x_{0}\right)=\left\{e^{t_{k} f_{k}} \circ \cdots \circ e^{t_{1} f_{1}}\left(x_{0}\right) \mid k \in \mathbb{N}, t_{1}, \ldots, t_{k} \in \mathbb{R}, f_{1}, \ldots, f_{k} \in \mathcal{F}\right\} .
$$

Remark 33. $\mathcal{O}\left(x_{0}\right)$ can be interpreted as the reachable set, starting from $x_{0}$, of the family $-\mathcal{F} \cup \mathcal{F}$, using only piecewise constant controls.

We have the following result 
Theorem 34 (Orbit theorem). For every $x_{0} \in \mathbb{R}^{n}$, the set $\mathcal{O}\left(x_{0}\right)$ has the structure of an immersed sub-manifold of $\mathbb{R}^{n}$. In particular it has the same dimension at every point. Moreover if $x \in \mathcal{O}\left(x_{0}\right)$ then $\operatorname{Lie}_{x}(\mathcal{F}) \subseteq T_{x} \mathcal{O}\left(x_{0}\right)$. The two spaces $\operatorname{Lie}_{x}(\mathcal{F})$ and $T_{x} \mathcal{O}\left(x_{0}\right)$ coincide if one of the following two conditions is verified:

- every element of $\mathcal{F}$ is an analytic vector field;

- the dimension of $\operatorname{Lie}_{x}(\mathcal{F})$ is constant with respect to $x \in \mathcal{O}\left(x_{0}\right)$.

From the fact that $\operatorname{Lie}_{x}(\mathcal{F}) \subseteq T_{x} \mathcal{O}\left(x_{0}\right)$ it follows that every element of $F$ is tangent to $\mathcal{O}\left(x_{0}\right)$. As a consequence we have the following.

Lemma 35. For every $x_{0} \in \mathbb{R}^{n}$ we have that $\mathcal{A}\left(x_{0}\right) \subset \mathcal{O}\left(x_{0}\right)$.

This result wouldn't be so obvious without the orbit theorem since $\mathcal{A}\left(x_{0}\right)$ is the set of points that one can reach using $L^{\infty}$ controls (and not only piecewise constant ones).

The following corollary gives some consequence of the Orbit theorem on the controllability of nonlinear systems.

Corollary 36. If $\mathcal{F}$ is not Lie bracket generating and either every vector field of $\mathcal{F}$ is analytic or the dimension of $\operatorname{Lie}_{x}(\mathcal{F})$ is constant with respect to $x \in \mathcal{O}\left(x_{0}\right)$, then $\mathcal{F}$ is not controllable.

Acknowledgements: This work was supported by the ERC POC project ARTIV1 contract number 727283, by the ANR project "SRGI" ANR-15- CE40-0018, by the ANR project "Quaco" ANR-17-CE40-0007-01 and by a public grant as part of the Investissement d'avenir project, reference ANR-11-LABX-0056-LMH, LabEx LMH (in a joint call with Programme Gaspard Monge en Optimisation et Recherche Opérationnelle).

\section{References}

[1] A. A. Agrachev and Y. L. Sachkov. Control theory from the geometric viewpoint, volume 87 of Encyclopaedia of Mathematical Sciences. Springer-Verlag, Berlin, 2004. Control Theory and Optimization, II.

[2] F. Jean. Control of nonholonomic systems: from sub-Riemannian geometry to motion planning. SpringerBriefs in Mathematics. Springer, Cham, 2014.

[3] V. Jurdjevic. Geometric control theory, volume 52 of Cambridge Studies in Advanced Mathematics. Cambridge University Press, Cambridge, 1997. 